\section{Tramadol Hydrochloride: A Prospective Local Anesthetic Agent}

\section{Abstract}

Local anaesthesia has been widely practiced with minimal risk of complications. To evaluate the local anesthetic property of $5 \%$ tramadol hydrochloride with adrenaline, a prospective research study was conducted in the Oral and Maxillofacial Surgery unit of Bokaro General Hospital, Jharkhand. A total number of 39 patients who needed various oral and maxillofacial surgical procedures were selected as per the inclusion and exclusion criteria. Pain on injection, onset of anesthesia, volume of $5 \%$ tramadol hydrochloride used, duration of anesthesia and post-operative analgesia was assessed for the same. Study showed that $5 \%$ Tramadol $\mathrm{HCl}$ with adrenaline is a good alternative anaesthetic agent for local anaesthesia in oral and maxillofacial surgeries. Its dual property as an analgesic and anaesthetic could be advantageous for long-term surgical operations.

Keywords: Tramadol hydrochloride; Local anesthesia; Minor surgeries

Received: February 20, 2019; Accepted: March 06, 2019; Published: March 14, 2019

\section{Srivastava M*, Chowdhury S and Vishal $\mathrm{G}^{2}$}

\author{
Bokaro General Hospital, Bokaro Steel City, \\ Jharkhand, India \\ * Corresponding author: \\ Dr. Madhumita Srivastava
}

vaanya.vishal.10@gmail.com

Specialist, Oral and Maxillofacial Surgeon, Bokaro General Hospital, Bokaro Steel City, Jharkhand, India.

Tel: 06542232750

Citation: Srivastava M, Chowdhury S, Vishal G (2019) Tramadol Hydrochloride: A Prospective Local Anesthetic Agent . Insights Biomed Vol.4 No.1:8

\section{Introduction}

Pain was defined in 1906 in The Devil's Dictionary as "an uncomfortable frame of mind that may have a physical basis in something that is being done to the body or may be purely mental. The most common method for blocking pain during oral surgical procedures is the intraoral administration of local anesthetic agent $[1,2]$. Tramadol hydrochloride $(\mathrm{HCl})$, a centrally acting opioid analgesic, was synthesized in 1962 by Grunenthal $\mathrm{GmbH}$ in Germany and was made available to use for pain management in Germany since 1977. Clinically it is effective for the treatment of moderate to severe pain such as, postsurgical pain, obstetric pain, terminal cancer pain and pain of coronary origin. Local anesthetics are agents that reversible interfere with neural conduction and are widely used to provide pain control in surgical treatments. Pang and colleagues in 1998 for the first time reported on the anaesthetic property of commercially available tramadol when injected intradermally [3]. From then to now tramadol has been widely studied for its anesthetic property. Earlier studies used tramadol as anesthetic agent only for soft tissue procedures, until Yaha al Haideri used it for tooth extraction as an infiltrative anesthesia [4]. Thahani used it for nerve blocks for surgical extraction of impacted tooth and periapical surgery. In this present study we will be again evaluating its local anaesthetic efficacy in various oral and maxillofacial procedures.

\section{Aim and Objectives}

To evaluate anesthetic efficacy of $5 \%$ tramadol hydrochloride with $0.0225 \mathrm{mg}$ adrenaline in various oral and maxillofacial surgical procedures when used as a nerve block. Also, to evaluate incidence of allergic reaction, onset, duration and potency of anaesthesia, intraoperative pain and postoperative analgesia of tramadol as a local anesthetic agent.

\section{Materials and Methods}

To evaluate the local anesthetic potency of $5 \%$ tramadol hydrochloride a prospective research study was conducted in the Oral and Maxillofacial Surgery unit of Bokaro General Hospital, Jharkhand. A total number of 39 patients who needed various oral and maxillofacial surgical procedures were selected as per the inclusion and exclusion criteria. All patients were selected randomly irrespective of gender, caste, creed and religion. Patient ages were 18-50, out of which 19 male and 20 females were there. This study was initiated after obtaining approval from the ethical committee and detailed written informed consent of patients was procured. The surgical procedure done was described in Table 1. Material used for nerve block we need $5 \%$ tramadol hydrochloride and adrenalin $0.0125 \mathrm{mg}$. Nerve block of above-mentioned 
solution was used for various minor oral surgical procedures. Different block given were inferior alveolar, lingual, long buccal, mental and local infiltration (if required) given. Detail surgical procedures of various minor surgeries were as follows:

\section{Surgical disimpaction}

A total number of fifteen mandibular impacted teeth were selected in whom bone guttering was required. After achieving profound anesthesia incision was given as per desired tooth position, full thickness mucoperiosteal flap was raised and underlying bone was exposed. Required bone guttering was done with the help of micromotor, tooth was extracted, socket irrigated, and flap was close with sutures. Total operating time was 45 minutes for each case.

\section{Radicular cyst enucleation}

Total ten cases of radicular cyst were done. All cyst located in mandible were selected. Among these 2 cysts was associated with third molar, 1 with first molar and 10 with mandibular anterior. After planned incision, full thickness mucoperiosteal flap was raised and underlying bone was exposed. Causative tooth was extracted, and cyst was enucleated, making sure that all of the cystic lining has been removed. The enucleated cyst was sent for histopathological examination. Total operating time was 45 minutes for each case. Average operating time was 45 minutes.

\section{Biopsy}

Total six biopsies were done under local anesthesia. Lesions located either on lower buccal vestibule or buccal mucosa was selected. Among six cases four excisional and two incisional biopsies were there. Excised biopsy tissue was sent for histopathological examination. Average operating time was 15 minutes.

\section{Excision and reconstruction of premalignant lesions}

Four cases of excision and reconstruction premalignant lesion were done under local anesthesia. Five cases were of leukoplakia and two of lichen plannus. Complete excision of lesion was done and reconstructed with collagen. Excised lesion was sent for histopathological examination. Average operating time was 40 minutes.

\section{Scar revision}

Four cases of scar revision were done. All of them were post traumatic scar. Average operating time was 30 minutes for each case. Evaluation criteria were pain on injection, onset of anaesthesia, pin prick grading, local tissue reaction, total volume of anesthetic required to produce satisfactory anaesthesia, duration of the surgery, duration of anaesthesia, need for first analgesic, noninvasive vital data monitor, systemic adverse reaction and a $10-\mathrm{cm}$ visual analogue scale (VAS) was used to assess pain.

\section{Observations and Results}

A total number of 39 patients were included in this research study. Out of 39, 16 were male and 23 females. Age of patients ranged from Minimum 18 to Maximum 60 years (Table 2).

Pain on injection was scored as $0=$ No pain; $1=$ Mild pain; 2=Moderate pain; 3=Severe pain. Association between dental procedure performed and pain during local anesthetic injection showed that, 30 patients had no pain or mild pain and 9 patients had moderate to severe pain. Differentiation factor was 4 with the $p$-value of 0.98 . Onset of anesthesia maximum recorded was 4 minutes and minimum 2 minutes. According to chi square test value was 3.53 and differentiation factor was 4 (Table 3).

Association between minor surgical procedure performed and volume of tramadol hydrochloride required for anesthesia was significant for radicular cyst enucleation, i.e., out of ten; seven patients needed $4 \mathrm{ml}$ of local anesthetic (Figure 1). When measured pain on VAS scale only $12 \%(n=5)$ patients experienced pain that too mild during the operative procedure, rest $88 \%$ $(\mathrm{n}=34)$ had no pain throughout procedure (Figure 2).

Duration of the surgery minimum recorded was 15 minutes for biopsy and maximum for enucleation of radicular cyst 45 minutes. Duration of anesthesia minimum recorded was 60 minutes and maximum were 180 minutes. Maximum patients experienced anesthesia for one hour only. Out of 39 patients, 9 patients ( 7 of radicular cyst enucleation and 2 of surgical disimpaction) needed post-operative analgesia that to after 10 hours. Only one patient of surgical disimpaction needed analgesic just after 30 minutes (Table 4).

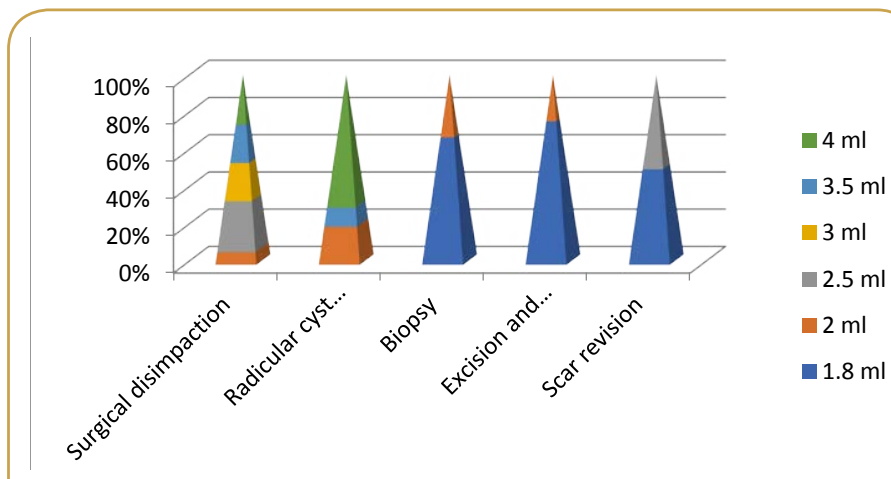

Figure 1 Association between dental procedure performed and volume of local anesthetic agent used.

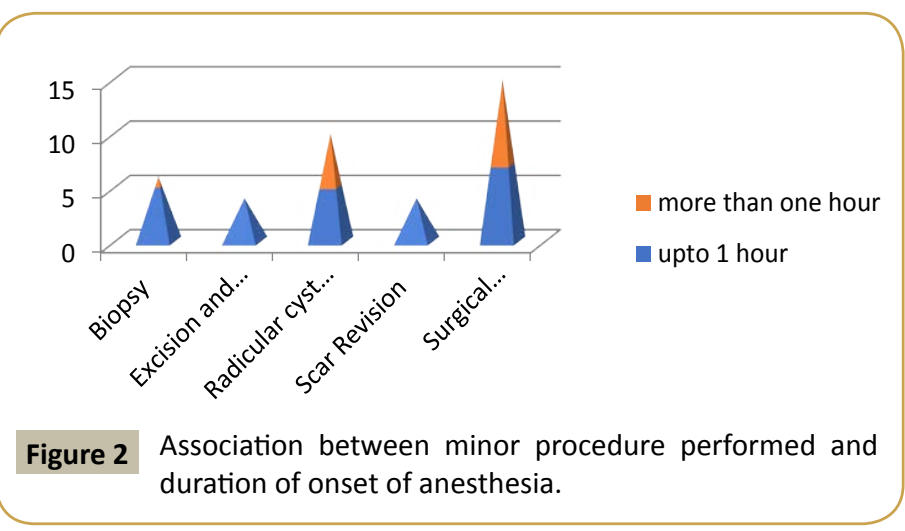


Table 1 Surgical procedures.

\begin{tabular}{ccc} 
Surgical Procedure Name & Number of Cases \\
Surgical disimpaction & 15 \\
Radicular cyst enucleation & 10 \\
Biopsy & 6 \\
Scar revision & 4 \\
\hline Excision and reconstruction of premalignant lesions & 4 \\
\hline
\end{tabular}

Table 2 Surgical procedures performed

\begin{tabular}{|c|c|c|c|}
\hline S. No & Procedure Performed & No. of Patients \\
\hline 1 & Biopsy & 6 \\
\hline 2 & Excision and Reconstruction of premalignant lesion & 4 \\
\hline 3 & Radicular cyst enucleation & 10 \\
\hline 4 & Scar Revision & 4 \\
\hline 5 & Surgical Impaction & 15 \\
\hline
\end{tabular}

Table 3 Surgical procedures performed with duration of onset of anesthesia.

\begin{tabular}{|c|c|c|}
\hline \multirow{2}{*}{ Procedure Performed } & \multicolumn{2}{|c|}{ Duration of Onset of Anesthesia } \\
\hline & Up to 2 minutes $(n=31)$ & More than 2 minutes $(n=8)$ \\
\hline Biopsy & $6(19.4)$ & 0 \\
\hline Excision and Reconstruction of premalignant lesion & $3(9.7)$ & $1(12.5)$ \\
\hline Radicular cyst enucleation & $7(22.6)$ & $3(37.5)$ \\
\hline Scar Revision & 4 (12.9) & 0 \\
\hline Surgical Impaction & $11(35.5)$ & $4(50.0)$ \\
\hline
\end{tabular}

Table 4 Surgical procedures performed with pain during procedure after local anesthesia.

\begin{tabular}{|c|c|c|}
\hline \multirow{2}{*}{ Procedure Performed } & \multicolumn{2}{|c|}{ Pain During Procedure After Local Anesthesia } \\
\hline & No pain $(n=34)$ & Slight Pain $(n=5)$ \\
\hline Biopsy & $6(17.6)$ & 0 \\
\hline Excision and Reconstruction of premalignant lesion & $3(8.8)$ & $1(20.0)$ \\
\hline Radicular cyst enucleation & $9(26.5)$ & $1(20.0)$ \\
\hline Scar Revision & $4(11.8)$ & 0 \\
\hline Surgical Impaction & $12(35.3)$ & $3(60.0)$ \\
\hline
\end{tabular}

\section{Discussion}

It is quite common in individuals to avoid surgical procedures, especially in oral and facial region because of fear of pain. Therefore, it is important to relieve the patient's anxiety during treatment by using a strong anesthetic in combination with agents that can have analgesic effects. The objective of the current study was to evaluate incidence of onset, duration and potency of anaesthesia, intraoperative pain and postoperative analgesia of $5 \%$ tramadol hydrochloride as a local anesthetic agent in minor surgical procedures.

At the $28^{\text {th }}$ WHO meeting of the Committee in 1992 tramadol hydrochloride was reviewed for the first time and on further reviewing process significant numbers of cases of withdrawal syndrome and dependence reported as adverse drug reactions were stated. In due course of time it was again reviewed at the 34 th meeting in 2006. Considering that tramadol continued to show a low level of dependence Committee concluded that there was not sufficient evidence to justify a critical review [5]. Compared to the classical opioid analgesic morphine and tramadol are considered to be a relatively safe analgesic. Few cases of fatal poisoning due to tramadol alone have been reported in the literature [6]. More frequent are intoxications with co-ingestion of other drugs or alcohol [7]. Intravenous naloxone has been successfully used to reverse the opioid effects of tramadol overdose [5].

Tramadol $\mathrm{HCL}$ is a synthetic centrally acting opioid; it is clinically effective for the treatment of moderate to severe pain with a relative low addiction incidence. In acute therapeutic use, tramadol $\mathrm{HCL}$ produces analgesia against multiple pain conditions such as postsurgical pain, obstetric pain, terminal cancer pain, and pain of coronary origin, and it has been used as adjuvant therapy in anesthesia. Due to its strong analgesic effect in medicine, tramadol has been used very commonly as an analgesic in dentistry. In 2015 tramadol $\mathrm{HCl}$ was administered in the sub mucosa for third molar extractions postoperatively and was found to be more effective at preventing acute pain [8]. 
Pozos et al. added tramadol $50 \mathrm{mg} / \mathrm{mL}$ to increase the efficacy of $4 \%$ articaine with 1:100,000 epinephrine to provide mandibular anaesthesia in third molar extraction, and they observed a synergistic effect [9]. Similar effects were seen in a study done by De Pedro-Munoz and Mena-Alvarez in 2017 [10]. In contrast when Ceccheti et al. used 2\% mepivacaine with 1:20,000 levonordefrin for inferior alveolar nerve anaesthesia in third molar extraction and postoperatively infiltrated tramadol $100 \mathrm{mg}$ sub mucosal immediately after extraction, they inferred that tramadol didn't increase anaesthetic efficacy although effective analgesia was achieved [11].

The recommended daily dose of tramadol is $100-400 \mathrm{mg}$. Maximum dose of tramadol for a $70 \mathrm{~kg}$ adult is $400 \mathrm{mg} /$ day $(5.7$ $\mathrm{mg} / \mathrm{kg}$ ). Each $2 \mathrm{ml}$ ampoule contains tramadol hydrochloride $100 \mathrm{mg}$. The distribution volume of tramadol is about 2.6-2.9 $\mathrm{L} / \mathrm{kg}$ bodyweight, following a $100 \mathrm{mg}$ intravenous dose. Plasma protein binding is approximately $20 \%$.

Human data shows that tramadol produces opioid-like effects when taken orally, but not after parenteral administration. In the last few years, new data have been reported and these data confirm that tramadol dependence may occur when used daily for more than a few weeks/months [5]. In our study we have used tramadol as a nerve block.

Study indicated that tramadol plus adrenaline is an effective method to reduce the post-operative pain, operative time and time to achieve haemostasis in tonsillectomy surgeries. Adrenaline which is the commonly used topical haemostatic agent is a powerful vasoconstrictor producing retraction of blood vessels in the tonsillar bed. Adrenaline promotes platelet aggregation in the formation of blood clot and has been demonstrated by Hatton to be inexpensive, posing little risk, and decreasing intraoperative bleeding, therefore can be taken as a reasonable haemostatic agent. Less intra operative bleeding leads to less use of cautery to achieve haemostasis and it added to comparatively less post-operative pain [12]. In our present study along with less intraoperative bleed and postoperative pain, addition of adrenaline also elongated the time of local anesthesia.

It has been demonstrated that tramadol hydrochloride $5 \%$ has a local anesthetic effect similar to that of prilocaine $2 \%$ when used intradermally for excision of soft tissue lesions $[13,14]$. Other studies showed that tramadol HCL has a local anesthetic activity similar to, but weaker than that of lidocaine $\operatorname{HCL}[15,16]$. Our present study we also evaluated the local anesthetic effect of tramadol hydrochloride, but it should be noted that the present study involved soft tissue incision and bone removal while other studies involved only soft tissue surgery.

Tramadol HCL may have local anesthesia like activity because of its nerve conduction blocking potency. Clinical and laboratory studies of opioids, such as tramadol, have shown that local anesthetic effects of opioids typically cannot be reversed by pretreatment with naloxone. Lack of antagonizing ability of naloxone is considered to be obvious because the effects of tramadol $\mathrm{HCL}$ on the nerve conduction are more likely mediated by a non-opioid receptor mediated mechanism. In addition, it has been reported that there is no competition for a common binding site on the $\mathrm{Na}$ channel between opioid, meperidine, and the local anesthesia lidocaine $[17,18]$. In clinical studies, it has been postulated that, it has a local anaesthetics effect similar to lidocaine following intradermal injections.

Immediately after injection (considered as time zero) to the time that the patient feels paresthesia on the respective area, supplied by specific nerve was recorded to be the onset of anesthesia. Paresthesia was checked both via subjective and objective symptoms. In our present study onset of anesthesia maximum recorded was 4 minutes and minimum 2 minutes. Onset of anesthesia for tramadol was also checked by Altunkaya et al. and Tahani Alsandook pilot study, where they found no significant difference when compared with prilocaine and lidocaine respectively [19,20]. Farnad Imani et al. in 2011 also inferred that when tramadol was added in $2 \%$ lidocaine in the epidural anesthesia for caesarean sections, sensory and motor blockades was more rapidly achieved [21].

The maximum volume of anesthetic used was $4 \mathrm{ml}$ in enucleation of radicular cyst and minimum required was $1.8 \mathrm{ml}$. Study done by Yahya, Al-Haideri and Tahani Alsandook in also did not show significant volume of anesthetic used, i.e., similar volume of either tramadol anesthetic is required for anesthetizing is used. In our present study degree of pain was evaluated with a $10 \mathrm{~cm}$ visual analog scale (VAS). Only 5 patients felt pain during procedure that too very mild. Painless surgical procedures using tramadol as a local anesthetic agent were also performed earlier by Altunkaya et al., Vahabi et al., Tahani Alsandook, Kemal Varım Numanog $ॅ$ lu and many more $[13,22,23]$.

\section{Conclusion}

Reviewing its analgesic property, tramadol when used as a local anesthetic agent provided the best analgesia. During study only one patient of surgical disimpaction needed analgesic just after 30 minutes, 9 patients after 10 and rest all didn't take analgesic within 24 hours. In terms of duration of local anesthesia for minor surgical procedures, tramadol provided adequate time. Although the feeling of numbness and heaviness which is considered a disadvantage due to discomfort associated with local anesthesia was of less duration. Hence there is also no need for local anesthetic reversal medication. $5 \%$ Tramadol $\mathrm{HCl}$ with adrenaline is a good alternative anesthetic agent for local anesthesia in oral and maxillofacial surgeries. Its versatile effect as an analgesic and anesthetic could be advantageous for long-term surgical operations, considering its substantial analgesic activity. 


\section{References}

1 Bridenbaugh PO (2002) Management of pain and anxiety in the dental office.

2 OMFS Clinics of North America (2010) Determining the appropriate oral surgery anesthesia modality, setting and team.

3 Pang WW, Mok MS, Chang DP, Huang MH (1998) Local anesthetic effect of tramadol, metoclopramide, and lidocaine following intradermal injection. Reg Anesth Pain Med 23: 580.

4 Yahya A, Al-Haideri A (2013) Comparison of local anesthetic efficacy of tramadol hydrochloride with adrenaline versus plain tramadol hydrochloride in the extraction of upper molar teeth. J Oral Maxillofac Surg 1: 1-2.

$536^{\text {th }}$ ECDD (2014) Agenda item 6.1

6 De Backer B, Renardy F, Denooz R, Charlier C (2010) Quantification in post-mortem blood and identification in urine of tramadol and its two main metabolites in two cases of lethal tramadol intoxication. $J$ Anal Toxicol 34: 599-604.

7 Clarot F, Goulle JP, Vaz E, Proust B (2003) Fatal overdoses of tramadol: Is benzodiazepine a risk factor of lethality? Forensic Sci Int 134: 5761.

8 G€on€ul O, Satilmıs T, Bayram F (2015) Effect of sub-mucosal application of tramadol on postoperative pain after third molar surgery. Head Face Med 11: 35.

9 Pozos AJ, Martinez R, Aguirre P (2006) The effects of tramadol added to articaine on anesthesia duration. Oral Surg Oral Med Oral Pathol Oral Radiol Endod 102: 614.

10 De Pedro-Munoz A, Mena-Alvarez J (2017) The effect of preoperative submucosal administration of tramadol on the success rate of inferior alveolar nerve block on mandibular molars with symptomatic irreversible pulpitis: A randomized, double-blind placebo-controlled clinical trial. Int Endod J 50: 1134.

11 Ceccheti MM, Negrato GV, Peres MP (2014) Analgesic and adjuvant anesthetic effect of submucosal tramadol after mandibular third molar surgery. Oral Surg Oral Med Oral Pathol Oral Radiol 117: e249.

12 Beigh Z, Islam M, Ahmad S, Pampuri RA (2013) Local anaesthetic effects of tramadol. Iranian J Otorinolaryngol 25: 72.
13 Altunkaya H, Ozer Y, Kargi E, Babuccu O (2003) Comparison of local anaesthetic effects of tramadol with prilocaine for minor surgical procedures. British J Anaes 90: 320.

14 Kargi E, Babuccu O, Altunkaya H, Hosnuter M, Ozer Y, et al. (2008) Tramadol as a local anaesthetic in tendon repair surgery. Hand J Int Med Res 36: 971.

15 Mert T, Gunes Y, Guven M, Gunay I, Ozcengiz D (2009) Blocking action of tramadol on nerve conduction 2001

16 Tufan M, Yasemin G, Ismail G (2006) Comparative effects of lidocaine and tramadol on injured peripheral nerves. Eur J Pharmacol 543: 54 62.

17 Yu-Chuan T, Pei-Jung C, Ming J (2001) Direct Tramadol application on sciatic nerve inhibits spinal somatosensory evoked potentials in rats. Anesth Analg 92: 1547-1551.

18 Brau ME, Koch ED, Vogel W (2000) Tonic blocking action of meperidine on $\mathrm{Na}^{+}$and $\mathrm{K}^{+}$channels in amphibian peripheral nerves. Anesthesiol 92: 147.

19 Altunkaya H, Ozer Y, Kargi E, Babuccu O (2003) Comparison of local anaesthetic effects of tramadol with prilocaine for minor surgical procedures. British J Anaes 1: 1-2.

20 Tahani A, Yahya A, Al-Haideri A (2013) A pilot double blinded clinical trial to compare between Tramadol $\mathrm{HCL}$ and Lidocaine $\mathrm{HCL}$ as local anaesthesia amongst hospital-outpatient adult dental attendees Mosul-Iraqi. J Oral Dental Res 1: 18-22.

21 Imani F, Entezary SR, Alebouyeh MR, Parhizgar S (2010) The maternal and neonatal effects of adding tramadol to $2 \%$ lidocaine in epidural anesthesia for cesarean section. Anesth Pain 1: 25-29.

22 Vahabi S, Heidari M, Ahmadinejad M, Akhlaghi J, Birjandi M (2011) Comparison of local anesthetic effects of Tramadol and Lidocaine used subcutaneously in minor surgeries with local anesthesia. Anesth 21: 1-2.

23 Kemal V, Hilal A, Duygu T, Ebubekir E (2014) Efficacy of tramadol as a preincisional infiltration anesthetic in children undergoing inguinal hernia repair: A prospective randomized study. Therap Clin Risk Manag 10: 753-758. 